\section{E-058 PILOT STUDY: FEASIBILITY OF THE USE OF VENTURA EMERGENCY LARGE VESSEL OCCLUSION SCALE TO IDENTIFY LARGE VESSEL OCCLUSION STROKE}

M Taqi, S Quadri, S Suriya. California Institute for Neuro-Innovations, Thousand Oaks, CA

10.1136/neurintsurg-2016-012589.130

Objectives To evaluate the feasibility and reliability of a selfdesigned Ventura Emergency Large Vessel Occlusion (ELVO) Scale performed by Emergency Medical Services (EMS). To identify, if field identification of ELVO, reduces door to groin time.

Background Endovascular treatment of stroke is now the standard of care for large vessel occlusion (LVO) stroke within 6 hours of onset. The outcomes of intra-arterial (IA) endovascular treatment are time dependent. The time from arrival to groin vary during the day time versus after hours or infield transfers compared to hospital to hospital transfers. Pre-identification of LVO from field can shorten the door to groin time by activation of Interventional Radiology (IR) team earlier. We designed a scale that can be used by EMS personnel in the field to identify ELVO or non-Emergency Large Vessel Occlusion.

Methods and analysis Ventura ELVO Scale (VES) comprise of four components: 1) Eye Deviation 2) Aphasia 3) Neglect 4) Obtundation, each component scoring either 1 or 0 . The maximum score is 4 and minimum score is 0 . The score of 1 or greater will be considered as ELVO positive. VES will be implemented by EMS to identify ELVO patients in the catchment area of Los Robles Hospital and Medical Center. Retrospective data will be collected from EMS, Emergency Department (ED) and IR records of participating patients. Door to groin time for intra-arterial (IA) treatment before implementation of the Ventura ELVO protocol will be compared with door to groin time after implementation of Ventura ELVO protocol. In prospective patients, EMS will measure ELVO score in the field using the VES. A positive ELVO score along with positive Cincinnati scale will prompt ELVO activation. EMS will call the neuro-interventionist who will then activate the neuro-intervention protocol at the receiving Hospital.

Implications Ventura ELVO scale can help to reduce door to groin puncture time, which can possibly improve the management and triage of stroke patients with ELVO.

Disclosures M. Taqi: None. S. Quadri: None. S. Suriya: None.

\section{E-059 BLEOMYCIN SCLEROTHERAPY FOR EYELID VENOUS MALFORMATIONS AS AN ALTERNATIVE TO SURGERY OR LASER THERAPY}

A Berenstein. Neurosurgery, Mount Sinai Health System, New York, NY

10.1136/neurintsurg-2016-012589.131

Purpose To describe the use of bleomycin in a foam preparation and or radiopaque, and or undiluted in slow flow venous malformations that involve the mucosa of the eyelid, and periorbital area.

Material and methods 11 patients with venous malformations, involving the mucosa of the eyelid, and periorbital area, were treated with various combination bleomycin injections in to the lesion under direct puncture, and monitored with US and/ or DSA. We will describe the various techniques and when best to use them.

Results There was significant improvement, or near total resolution in all patients with a follow up of up to 4 years, there were 2 recurrences, there were no complications

Conclusions The use of bleomycin in various forms appears as a simple, safe and very effective treatment for venous malformations involving the mucosa of the eyelid, and periorbital area, avoiding more elaborated and challenging surgical or laser interventions, and is effective in thicker lesions.

Disclosures A. Berenstein: None.

\section{E-060 COIL EMBOLIZATION OF SMALL AND WIDE NECKED INTRACRANIAL ANEURYSM WITH TEMPORARY STENT ASSISTED TECHNIQUE}

W Yoon. Neurosurgery, The Catholic University of Korea, Seoul, Republic of Korea

\subsection{6/neurintsurg-2016-012589.132}

Purpose For small and wide-necked intracranial aneurysms, the authors present a novel temporary stent-assisted technique (TemSAT) using fully retrievable stent, the Solitaire AB.

Materials and methods Data of a small series of patients who underwent TemSAT was prospectively collected and analyzed. Total 12 patients with 12 aneurysms were enrolled from March 2011 to May 2011. The basic characteristics of the aneurysms, radiologic and clinical outcome were analyzed.

Results Five p-com, 4 paraclinoid ICA, 1 a-com, 1 MCA bifurcation and $1 \mathrm{~V} 4$ aneurysms were treated with this technique. One of the p-com aneurysm was ruptured and the other aneurysms were unruptured. Their size was small less than 10 $\mathrm{mm}$ (2.5-9.0 mm). The mean aspect ratio was 1.23 (1.1-1.4). Immediate angiographic results were complete in 11 cases and neck remnant in 1 case. There were 2 cases of intraprocedural in-stent thrombosis complication, which resolved after removal of the stent and intra-arterial thrombolytic agent injection. There was no cases of postoperative neurologic complications. The average packing density of the aneurysm was $33.9 \%$ (19.9-57.3).

Conclusion By temporary neck remodeling with retrievable stent, blood flow obstruction risk could be reduced contrary to balloon assisted technique. In addition, there is no need for life-long antithrombotic agent administration. However, due to the characteristics of the Solitaire stent, natural angle of the artery was changed and it resulted in in-stent thrombosis. This technique can be effective in carefully selected cases. Abbreviations a-com anterior communicating artery MCA middle cerebral artery p-com posterior communicating artery V4 vertebral artery 4 th segment Disclosures W. Yoon: None.

\section{E-061 WHAT'S THE RELIABILITY AND SIGNIFICANCE OF PRE- COILING CT ANGIOGRAPHY IN RUPTURED CEREBRAL ANEURYSMS?}

J Sung, D Lee, S Kim, H Lee, S Lee. Department of Neurosurgery, St. Vincent's Hospital, The Catholic University of Korea, Suwon, Republic of Korea

10.1136/neurintsurg-2016-012589.133 